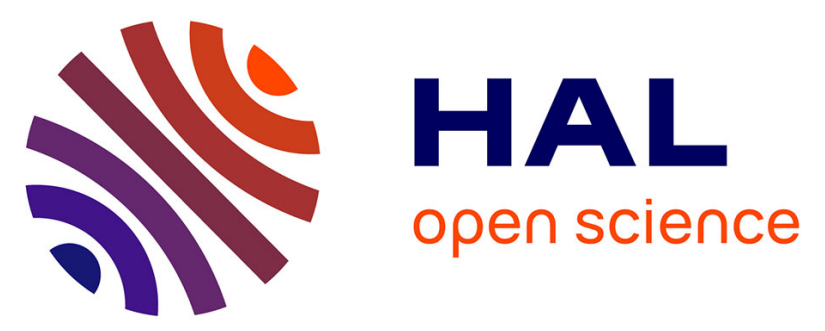

\title{
Characterisation of middle-distillates by comprehensive two-dimensional gas chromatography (GCxGC): A powerful alternative for performing various standard analysis of middle-distillates
}

Colombe Vendeuvre, Rosario Ruiz-Guerrero, Fabrice Bertoncini, Laurent

Duval, Didier Thiébaut, Marie-Claire Hennion

\section{To cite this version:}

Colombe Vendeuvre, Rosario Ruiz-Guerrero, Fabrice Bertoncini, Laurent Duval, Didier Thiébaut, et al.. Characterisation of middle-distillates by comprehensive two-dimensional gas chromatography (GCxGC): A powerful alternative for performing various standard analysis of middle-distillates. Journal of Chromatography A, 2005, 2nd International Symposium on Comprehensive Multidimensional Gas Chromatography, 2nd International Symposium on Comprehensive Multidimensional Gas Chromatography, 1086 (1-2), pp.21-28. 10.1016/j.chroma.2005.05.106 . hal-01330596

\section{HAL Id: hal-01330596 \\ https://hal-ifp.archives-ouvertes.fr/hal-01330596}

Submitted on 11 Jun 2016

HAL is a multi-disciplinary open access archive for the deposit and dissemination of scientific research documents, whether they are published or not. The documents may come from teaching and research institutions in France or abroad, or from public or private research centers.
L'archive ouverte pluridisciplinaire HAL, est destinée au dépôt et à la diffusion de documents scientifiques de niveau recherche, publiés ou non, émanant des établissements d'enseignement et de recherche français ou étrangers, des laboratoires publics ou privés. 
Ref. No : JCA-04-480

Characterisation of middle-distillates by comprehensive two-dimensional gas chromatography (GC×GC): A powerful alternative for performing various standard analysis of middle-distillates

Colombe Vendeuvre ${ }^{1}$, Rosario Ruiz-Guerrero ${ }^{1}$, Fabrice Bertoncini $^{1 *}$, Laurent Duval ${ }^{1}$, Didier Thiébaut $^{2}$, Marie-Claire Hennion ${ }^{2}$

1-Institut Français du Pétrole, BP3, 69390 Vernaison, France

2-ESPCI, Laboratoire Environnement et Chimie Analytique, 10 rue Vauquelin, 75235 Paris Cedex 05, France

*Corresponding author: fabrice.bertoncini@ifp.fr - Fax number: (33) 478022745 
Abstract

The detailed characterisation of middle distillates is essential for a better understanding of reactions involved in refining process. Owing to higher resolution power and enhanced sensitivity, comprehensive two-dimensional gas chromatography $(\mathrm{GC} \times \mathrm{GC})$ is a powerful tool for improving characterisation of petroleum samples. The aim of this paper is to compare GC $\times$ GC and various ASTM methods - gas chromatography (GC), liquid chromatography (LC) and mass spectrometry (MS) - for group type separation and detailed hydrocarbon analysis. Best features of $\mathrm{GC} \times \mathrm{GC}$ are demonstrated and compared to these techniques in terms of cost, time consumption and accuracy. In particular, a new approach of simulated distillation (SimDis-GC $\times \mathrm{GC}$ ) is proposed: compared to the standard method ASTM D2887 it gives unequal information for better understanding of conversion process.

Key words : comprehensive two dimensional gas chromatography, ASTM methods, simulated distillation, group type separation, hydrocarbons 


\section{Introduction}

The growing need of the European market in diesel fuel combined with the development of new refinery processes requires a deeper insight into the middle distillates $\left(150^{\circ} \mathrm{C}-400^{\circ} \mathrm{C}\right)$. Their characterisation, based on the boiling range distribution and the chemical composition, is currently achieved by different ASTM test methods. The group type analysis is performed either by liquid chromatography (LC) for the saturates/aromatics balance (ASTM D2549 [1]) or by mass spectrometry (MS) to determine the composition of the petroleum cut in saturate, monoaromatic, diaromatic and triaromatic hydrocarbons; for example ASTM D2425 [2] provides the repartition of hydrocarbons in 11 families. The boiling point distribution is obtained by gas chromatography (GC) using the so called simulated distillation (SimDis) method based on the fact that hydrocarbons are eluted from a non-polar column according to their boiling point $[3,4]$. Using a mixture of n-paraffins with known boiling points, the correspondence between the retention times and the boiling points is established and the cumulated weight percent versus the boiling point curve can be constructed. SimDis has become the major analytical tool for the characterization of petroleum products in research and refinery laboratories since it may advantageously replace conventional distillation methods for control of refining processes or for product specifications. ASTM D2887 [5] is the SimDis method advocated for middle distillates. As informations on group type composition and boiling range are essential and complementary, a method that would combine both determinations would be desirable.

Comprehensive two-dimensional gas chromatography $(\mathrm{GC} \times \mathrm{GC})$ has revealed a huge potential for investigating complex mixtures such as petroleum products owing to its improved resoluting power. Its principle and applications have recently been reviewed [6]. The wellknown polarity versus volatility separation achieved using a first classical non-polar column 
connected to a second fast semi-polar column leads to structured chromatograms where hydrocarbons are arranged according to their chemical group and to their number of carbon atoms. In the petroleum field, middle distillates - kerosene and diesel fuels - are probably the most interesting samples to be analysed in $\mathrm{GC} \times \mathrm{GC}$ : their complexity prevents their detailed analysis in conventional GC and their final boiling point is compatible with the maximum temperatures of columns. The characterisation of middle distillates by $\mathrm{GC} \times \mathrm{GC}$ was reported for qualitative [7] or quantitative [8] analyses. Although the Flame Ionisation Detector (FID) is the most appropriate detector for routine and quantitative analysis of hydrocarbons, hyphenation with a mass spectrometer [9] or specific detectors - atomic emission detector (AED) [10] or sulphur chemiluminescence detector (SCD) $[11,12]$ - is recommended for identification or speciation. Some studies compared $\mathrm{GC} \times \mathrm{GC}$ with other analytical techniques to ensure that reliable results were obtained. Frysinger et al. demonstrated the good agreement of GC $\times \mathrm{GC}$ results with ASTM test methods for the determination of BTEX [13] and oxygenates [14] in gasoline. Besides, it was also shown that the $\mathrm{GC} \times \mathrm{GC}$ group type separation of a gasoil correctly matched LC-GC results [8]. In this study, GC $\times \mathrm{GC}$ is demonstrated as a powerful tool to obtain the group type separation of a gasoil combined with its boiling range distribution. A comparison with ASTM methods is undertaken in order to validate this new approach.

\section{Experimental}

\subsection{Liquid Chromatography (LC)}

A column (diameter: $9.53 \mathrm{~mm}$ ) was filled with silica and alumina (Alumina 90) supplied by Merck (Darmstadt, Germany). After conditioning for 30 min under n-heptane flow, the sample $(0.5$ to $1 \mathrm{~g})$ diluted in $\mathrm{n}$-heptane was injected via a $4 \mathrm{ml}$ loop. The flow rate of the 
column was set at $2 \mathrm{ml} / \mathrm{min}$. The saturated compounds were eluted with $\mathrm{n}$-heptane for $37 \mathrm{~min}$ and the aromatic compounds were eluted with an n-heptane/toluene (70/30, v/v) mixture for 3 hours. The standard procedure of group type separation of petroleum products by LC was simplified for middle distillates, owing to the absence of resins and asphaltenes in these samples (only two elution steps). Each collected fraction was vaporised under nitrogen flow and the dried residue was weighed to determine the saturate/aromatic distribution.

\subsection{Mass spectrometry (MS)}

A mass spectrometry analysis method derived from ASTM D2425 and developed by Castex et al. [15] was used to obtain the composition of middle distillates. Its principle is based on the representation of a hydrocarbon family by the sum of fragments and molecular ions and on the calculation of the concentration from coefficient matrices depending on the average carbon number. Contrary to the D2425 method, the prior separation by liquid chromatography (ASTM D2549) was avoided owing to the operation at medium resolution ( $\mathrm{R}=5000)$, which allowed the separation of different families having the same entire mass. MS analysis was performed by electronic impact at $70 \mathrm{eV}$ using a high resolution spectrometer Ultima from Fisons (East Grinstead, UK). Some ten spectra were acquired at 3 s/decade with a 30-350 a.m.u. mass range and were averaged to improve the signal/noise ratio.

\subsection{GC Simulated Distillation (SimDis)}

Simulated distillation analysis was achieved using a HP5890 chromatograph (Agilent Technologies, Massy, France) equipped with a constant flow rate of carrier gas, a flame ionisation detector (FID) and a cool on-column inlet. A MXT-1 (Restek, France) Silcosteel®treated stainless steel capillary column $(15 \mathrm{~m} \times 0.53 \mathrm{~mm}$ i.d.; $0.5 \mu \mathrm{m})$ was heated from $35^{\circ} \mathrm{C}$ (hold $1 \mathrm{~min}$ ) to $390^{\circ} \mathrm{C}$ at a rate of $10^{\circ} \mathrm{C} / \mathrm{min}$. Helium flow rate was kept constant at $10 \mathrm{ml} / \mathrm{min}$. 
Detector temperature was set at $400^{\circ} \mathrm{C}$. The SimDis curve was obtained by using the algorithm defined in the ASTM D2887 method. The calculation was performed using the Chromdis software (Gecil Process, France).

\subsection{Comprehensive two-dimensional gas chromatography $(\mathrm{GC} \times \mathrm{GC})$}

GC $\times$ GC analyses were carried out using a HP6890 chromatograph (Agilent Technologies) equipped with a split/splitless injector and a FID. $\mathrm{A} \mathrm{CO}_{2}$ dual jets modulator was built in house as described by Beens et al. [16] and now provided as standard on the GC $\times \mathrm{GC}$ from ThermoElectron, and installed in the chromatograph. Two different set of columns were used: a classic non-polar/polar combination of columns for "normal phase" $\mathrm{GC} \times \mathrm{GC}(\mathrm{nP}-\mathrm{GC} \times \mathrm{GC})$ and a polar/non-polar system for "reversed phase" $\mathrm{GC} \times \mathrm{GC}(\mathrm{rP}-\mathrm{GC} \times \mathrm{GC})$. The two columns were placed in the same temperature programmed oven. Operating conditions are reported in Table 1. After acquisition using the ChemStation software (Agilent Technologies), data were processed by a Matlab program (The Mathworks, Natik, MA, USA) written in-house for twodimensional chromatogram visualisation and quantification. Other details are given in reference [17].

\subsection{Chemicals}

A synthetic mixture of hydrocarbons was prepared using chemical standards available at Sigma-Aldrich (Lyon, France). Normal paraffins were diluted in n-heptane and aromatic compounds in toluene, each in the range $350-500 \mathrm{ppm}$. The composition of the hydrocarbon mixture is given in Table 2. Air, helium and hydrogen were provided by Air Liquide (Feyzin, France) at a purity of $99.999 \%$. The petroleum sample was the gasoil cut $\left(213-347^{\circ} \mathrm{C}\right)$ obtained from straight run distillation of Safaniya crude oils purchased by IFP. They were injected neat. 
3. Results and discussion

\subsection{Group type separation}

\subsection{1. 'Normal Phase' GC $\times \mathrm{GC}(\mathrm{nP}-\mathrm{GC} \times \mathrm{GC})$}

An overview of the literature devoted to $\mathrm{GC} \times \mathrm{GC}$ indicates that the combination of a first nonpolar column with a second (semi)-polar column is very often used, especially for hydrocarbon analysis. This association leads to orthogonal separations as explained by Schoenmakers et al. [18], because two independent chemical properties - polarity and volatility - are involved in each of the two dimensions. This configuration, that we refer to as $\mathrm{nP}-\mathrm{GC} \times \mathrm{GC}$, was chosen to achieve the separation of a gasoil (Figure 1). Adapted operating conditions allowed separating saturate, mono-, di- and triaromatic hydrocarbons in four different bands owing to the different interaction of these compounds towards the stationary phase. Chromatograms were integrated by mouse-clicking the elution zones as represented in Figure 1. The definition of elution zones was based on retention times of chemical standards belonging to the different groups (Table 2) and could be adjusted by visualising peak start and peak stop symbolised by red/green crosses using a specific function similar to the one mentioned in reference [8].

\subsection{2. 'Reversed phase' $\mathrm{GC} \times \mathrm{GC}(\mathrm{rP}-\mathrm{GC} \times \mathrm{GC})$}

In this study, a reversed polarity set of columns, i.e. a first long polar column connected to a second short non-polar column, was also investigated to improve the separation between saturates and aromatics and, consequently, integration results. The $\mathrm{rP}-\mathrm{GC} \times \mathrm{GC}$ separation using conditions reported in Table 1 is presented in Figure 2. As a first observation, the separation space was extended compared to $\mathrm{nP}-\mathrm{GC} \times \mathrm{GC}$ separations meaning that the peak 
capacity was better adjusted to the analytical problem. The same feature was reported by Dimandja et al. [19] for the separation of standard compounds belonging to very different chemical classes. The structure of the chromatogram can be explained through retention considerations using the analogy with explanations proposed in reference [18] for the structure of $\mathrm{nP}-\mathrm{GC} \times \mathrm{GC}$ chromatograms. The retention of a compound $i$ is inversely proportional to its vapour pressure $\left(\mathrm{p}_{\mathrm{i}}{ }^{0}\right)$ and to its activity coefficient toward the stationary phase at infinite dilution $\left(\gamma_{\mathrm{i}}{ }^{0}\right)$. In a non-polar/polar system, two analytes, $i$ and $j$, having the same vapour pressure $\left(\mathrm{p}_{\mathrm{i}}{ }^{0}=\mathrm{p}_{\mathrm{j}}{ }^{0}\right)$ are coeluted after the first separation but may be separated in the second dimension on the basis of the difference in their activity coefficient $\left(\gamma_{i}^{0} \neq \gamma_{j}^{0}\right)$. In a polar/non-polar system, the first dimension separation is governed by both volatility and molecular specific interactions. This situation does not meet Giddings requirements for a multidimensional system because volatility is involved in both separation mechanisms [20]. However, analytes coeluted after the first separation $\left(\mathrm{p}_{\mathrm{i}}^{0} \gamma_{\mathrm{i}}^{0}=\mathrm{p}_{\mathrm{j}}^{0} \gamma_{\mathrm{j}}^{0}\right)$ may also be separated in the second dimension if their vapour pressures are different $\left(\mathrm{p}_{\mathrm{i}}{ }^{0} \neq \mathrm{p}_{\mathrm{j}}{ }^{0}\right)$, also meaning that they have different polarities $\left(\gamma_{i}^{0} \neq \gamma_{j}^{0}\right)$. This situation typically occurs for the separation between saturates and aromatics. In the first dimension, aromatics are coeluted with saturates of lower volatility $\left(\gamma_{\text {aromatic }}^{0}<\gamma_{\text {saturate }}^{0} ; \mathrm{p}_{\text {aromatic }}^{0}<\mathrm{p}_{\text {saturate }}^{0}\right)$. As the separation in the second dimension only depends on volatility, the aromatic compound has a lower retention time than the saturate compound introduced at the same time in the second column. This process is similar for the separation between mono-, di-, and triaromatics. In the second dimension of $\mathrm{rP}-\mathrm{GC} \times \mathrm{GC}$, compounds elute from the more to the less polar. The strength of $\mathrm{GC} \times \mathrm{GC}$ still relies on the possibility to resolve in a second separation two compounds that were coeluted after a first separation, owing to a difference in a specific chemical property. 
As in $\mathrm{nP}-\mathrm{GC} \times \mathrm{GC}$, a roof tile organisation was observed. A simple explanation based on retention index is proposed using three homologous alkylbenzenes: n-butylbenzene (NB), secbutylbenzene (SB), tert-butylbenzene (TB). In temperature programmed conditions, the linear alkylbenzene is more retained than the branched isomers because of two synergetic effects: a lower volatility and a better solubility in the polar stationary phase. The retention indexes of NB, SB and TB on a polar stationary phase (Carbowax 20M) are respectively 1307, 1242, 1231 [21]. The difference of retention index between linear and branched isomers (NB-SB) is greater when using a polar stationary phase than a non-polar one (OV1), respectively 65 and 45. Thus the difference in elution temperature of branched and linear isomers is greater in rP$\mathrm{GC} \times \mathrm{GC}$ than in $\mathrm{nP}-\mathrm{GC} \times \mathrm{GC}$. The linear isomer will be eluted at a higher temperature, resulting in a shorter second dimension retention time. This explains the orientation of tiles from the left top to the right bottom.

\subsubsection{Comparison of group-type separation with ASTM methods}

Using a FID, the response factors of saturate and aromatic hydrocarbons are approximately the same (within 5\%). Then, in a first approach, the area percentage calculated by integration of the $2 \mathrm{D}$ chromatograms is the same as the weight percentage. Raw integration data obtained for $\mathrm{n}$ replicates in $\mathrm{nP}-\mathrm{GC} \times \mathrm{GC}$ and in $\mathrm{rP}-\mathrm{GC} \times \mathrm{GC}$ correctly match $\mathrm{LC}$ and $\mathrm{MS}$ results for the saturates/aromatics balance (Table 3). A better agreement with conventional methods is observed in $\mathrm{rP}-\mathrm{GC} \times \mathrm{GC}$ probably because of a better separation between saturates and aromatics as it is shown in Figure 3 for the separation of hydrocarbons at the retention time of anthracene. This approach demonstrates that a change in column polarity is relevant for complex oil samples separation. Even if slight discrepancies occurred between LC, MS and $\mathrm{GC} \times \mathrm{GC}$ results, they can be regarded as similar considering that different methods involving different separation mechanisms were used to obtain these results for such complex mixtures. 
However, a better agreement would be expected if the discrimination at injection could be corrected. To take this effect into account, specific response factors were determined using the mixture of hydrocarbons whose composition is reported in Table 2. For each chemical group a relationship is established between the elution time, directly related to the number of carbon atoms when a non-polar column is used, and the response factor. Each group type band of the nP-GC $\times$ GC chromatogram was divided in slices whose width equals to the modulation period. A specific function of the Matlab program returns the area of each slice associated to a (first dimension) retention time. The area is then converted in a weight percent using the response factors. The correction of integration results indeed leads to a better agreement with MS (Table 3) : for example, the relative deviation calculated for saturates is $1.6 \%$. The correction of data in $\mathrm{rP}-\mathrm{GC} \times \mathrm{GC}$ does not appear as easy since the separation in the first dimension depends on the volatility and on the polarity. This points out the limits of hot split injection. The problem could have been circumvented by choosing an other type of system involving far less discrimination such as PTV injectors.

The features of these techniques are compared. LC is a time-consuming technique using large quantities of toxic and costly organic solvents. A loss of volatile compounds can occur during the evaporation step at the end of the procedure. Moreover, a minimum amount of $2 \mathrm{~g}$ of product is required, which is not compatible with the miniaturisation of process units (micropilots) that provide fewer quantities. Obviously, $\mathrm{GC} \times \mathrm{GC}$ analyses are far more rapid (80 min) and require less than $1 \mu \mathrm{l}$ of product. Comparing $\mathrm{GC} \times \mathrm{GC}$ with $\mathrm{MS}$, the necessary calibration step in MS to obtain quantitative results becomes a drawback when various samples have to be analysed. Moreover, ASTM D2425 is applicable to middle distillates with a boiling range limited to $204-343^{\circ} \mathrm{C}$ for 5 to $95 \%$ of their volume determined by laboratory distillation (ASTM D86). The relative content of triaromatics is then underestimated if the 
content of heavy hydrocarbons (b. p. $>350^{\circ} \mathrm{C}$ ) is non negligible. $\mathrm{GC} \times \mathrm{GC}$ gives the opportunity to extend the carbon range to minimum $450^{\circ} \mathrm{C}$, i.e. to a boiling point equivalent to $\mathrm{nC}_{30}$ (n-triacontane).

3.2. Detailed group type analysis: group type distribution versus the number of carbon atoms The structure of $\mathrm{GC} \times \mathrm{GC}$ chromatograms allows getting more detailed information on the sample composition than that provided by a simple group-type separation. Indeed, isomers are grouped in the same location of the chromatogram, and can be recognised owing to the rooftile effect [18]. The detailed group-type separation was obtained from the nP-GC $\times \mathrm{GC}$ chromatogram presented in Figure 1. Although individual peak resolution was still insufficient, elution zones of hydrocarbons of the same chemical family and with the same number of carbon atoms were plotted into clusters shown in Figure 4. The tricky definition of elution zones was partly confirmed by injection of standards listed in Table 2. Naphthenicaromatics were not integrated in a detailed way because isomer groups could not be defined with accuracy. Their quantification by the number of carbon atoms was not possible; however, they were quantified as a whole group to determine the weight content of other chemical groups.

The distribution in weight percent of hydrocarbons according to the chemical group type and to the number of carbon atoms is given in Figure 5; it is centred on $\mathrm{C}_{15}$ (hydrocarbons with 15 carbon atoms) for n-paraffins, mono and diaromatics, on $\mathrm{C}_{16}$ for naphthenic-diaromatics and triaromatics, and on $\mathrm{C}_{17}$ for isoparaffins. This kind of determination is rather new for middle distillates. Moreover, the $\mathrm{GC} \times \mathrm{GC}$ detailed separation offers the possibility to assign to each cluster of isomers a macroscopic property, such as the mass, the viscosity or the cetane number which can be known for each isomer group, resulting in more accurate information on 
physico-chemical properties of the gasoil cut. In one single analysis, the complete characterisation of the sample could be obtained and then directly compared to product specifications; a better modelling of kinetics and thermodynamics involved in refinery processes is also expected.

\section{3. $\mathrm{GC} \times \mathrm{GC}$ simulated distillation $(\mathrm{GC} \times \mathrm{GC}-\mathrm{SimDis})$}

The principle based on the conversion of the detailed information obtained by $\mathrm{GC} \times \mathrm{GC}$ into macroscopic properties was applied to determine the boiling range distribution of the gasoil. Using the data obtained for the quantification of each chemical group in $\mathrm{nP}-\mathrm{GC} \times \mathrm{GC}, \mathrm{GC} \times \mathrm{GC}$ simulated distillation curves could be constructed. Since chemical properties remain correlated in the first dimension of a polar/non-polar system, this configuration is not adapted to the simulated distillation approach presented here. The elution zone of one chemical group was divided into slices whose width equals to the modulation period. The retention time of the slice was converted into a boiling point using a relationship established between retention times and boiling points of standard compounds listed in Table 2. Then the area was converted into a cumulated weight percent using appropriate response factors and the total 'corrected' area of the two-dimensional chromatogram. As the signal returns to the baseline at each modulation cycle, it is not necessary to run a blank analysis as in conventional SimDis for subtracting the baseline drift. The conversion of retention times into boiling points raises some questions as it is well known that not all hydrocarbons elute from a non-polar column in their boiling point order (also meaning that $\mathrm{nP}-\mathrm{GC} \times \mathrm{GC}$ separations are not truly orthogonal). These problems were addressed in ASTM D2887. It was observed that deviations in SimDis from true boiling points (TBP) were $-11^{\circ} \mathrm{C}$ for naphthalene and $-35^{\circ} \mathrm{C}$ for phenanthrene. But the boiling points of standards in reduced pressure conditions were not so different than those obtained in SimDis. An acceptable explanation is that reduced pressure conditions are also 
encountered in some types of laboratory distillation (ASTM D1160); this justifies the calibration of SimDis curves with n-paraffins when comparing SimDis with these distillation methods. However, the process of a chromatographic separation is rather different from that of distillation as the stationary phase also plays a major role in the elution order. A decisive advantage of $\mathrm{GC} \times \mathrm{GC}$ simulated distillation is the possibility to use the TBP of compounds and thus specific scales of conversion for each separated band of chemical compounds. A calibration curve was plotted for each chemical family from retention data of standards whose location in the two-dimensional space covers the elution span of real samples. Unfortunately, TBP of alkylphenanthrenes or alkylanthracenes are not available. It was proposed to extrapolate them from the calibration curve of aromatic compounds and from the boiling points of anthracene and phenanthrene. One improvement of this procedure would consist in the determination of TBP of triaromatics from molecular simulation [22].

GC $\times$ GC-SimDis curves of saturates, mono-, di- and triaromatics are presented in Figure 6 . They represent the boiling point distribution of each chemical group according to the cumulated weight percent. The presence of normal paraffins which are the most concentrated products results in several flat parts in the SimDis curve of saturates. A high potential for characterising hydrotreatment process through the $\mathrm{GC} \times \mathrm{GC}-\mathrm{SimDis}$ analysis of the feed and the products is expected. The conversion of di- and triaromatics into naphthenic aromatics, and of aromatics into saturates is directly determined by the modification of SimDis curves: a precise information on the nature and the volatility of refractory and converted products is obtained. In order to validate these results, a comparison with conventional SimDis (ASTM D2887) was undertaken. A global SimDis curve was calculated in GC $\times$ GC from data used in Figure 6 and superimposed with the one determined in GC. As can be seen from Figure 7, an excellent agreement is found between both global determinations whose difference is lower 
than $1{ }^{\circ} \mathrm{C}$ for $10 \%$ to $90 \%$ of distilled product and lower than $2.5^{\circ} \mathrm{C}$ for the range $5-95 \%$. This validates and reinforces the $\mathrm{GC} \times \mathrm{GC}-\mathrm{SimDis}$ approach to analyse middle distillates.

\section{Conclusion}

Group type separation and simulated distillation results obtained by $\mathrm{GC} \times \mathrm{GC}$ are in line with ASTM methods based on LC, GC or MS; therefore they meet industrial requirements. Advantages over these conventional methods in terms of time and cost were outlined. The group type analysis per the number of carbon atoms and the determination of simulated distillation profiles for each chemical group were demonstrated as new possibilities offered by the technique to get a deeper insight in the molecular composition of middle distillates. Moreover, a different configuration based on column polarity inversion proved that group type separation can occur even if the orthogonality is not achieved. It opens up new opportunities to implement $\mathrm{GC} \times \mathrm{GC}$ in different configurations providing that a higher resolution is obtained.

\section{Acknowledgment}

N. Leymarie and I. Merdrignac are gratefully acknowledged for their contribution to this work. 


\section{References}

[1] American Society for Testing and Materials. ASTM D2549

[2] ASTM D2425. Annual book of ASTM Standards, Part.24, 367-374 (1980)

[3] F.T. Eggerston,S. Croennings, J.J. Holst, Anal. Chem. 32 (1960) 904

[4] L.E. Green, L.J. Schmauch, J.C. Worman, Anal. Chem. 36 (1964) 1512

[5] American Society for Testing and Materials. ASTM D2887 DS

[6] J. Dallüge, J. Beens, U. A.Th. Brinkman, J. Chromatogr. A, 1000 (2003) 69

[7] J. Blomberg, P. J. Schoenmakers, J. Beens, R. Tijssen, J. High Resol. Chromatogr. 20 (1997) 539

[8] J. Beens, H. Boelens, R. Tijssen, J. Blomberg, J. High Resol. Chromatogr. 21 (1998) 47

[9] M. van Deursen, J. Beens, J. Reijenga, P. Lipman, C. Cramers, J. High Resol.

Chromatogr. 23 (2000) 507

[10] L.L.P. van Stee, J. Beens, R.J.J. Vreuls, U.A.Th. Brinkman, J. Chromatogr. A 1019 (2003) 89

[11] R.Hua, Y. Li, W. Liu, J. Zheng, H. Wei, J. Wang, X. Lu, H. Kong, G. Xu, J. Chromatogr. A $1019(2003) 101$

[12] F.C.Y. Wang, W.K. Robbins, F.P. Di Sanzo, F.C. McElroy, J. Chromatogr. Sc. 41 (2003) 519

[13] G.S. Frysinger, R.B. Gaines, E. B. Ledford, J. High Resol. Chromatogr. 22 (1999) 195

[14] G.S. Frysinger, R.B. Gaines, J. High Resol. Chromatogr. 23 (2000) 197

[15] H. Castex, R. Boulet, J. Juguin, A. Lepinasse, Rev. Inst. franç. du pétr. 38 (1983) 523

[16] J. Beens, M. Adahchour, R.J.J. Vreuls, K. van Altena, U. A. Th. Brinkman, J. Chromatogr. A 919 (2001) 127

[17] C. Vendeuvre, F. Bertoncini, L. Duval, J.L. Duplan, D. Thiébaut, M.C. Hennion, J. Chromatogr. A, 1056 (2004) 155 
[18] P.J. Schoenmakers, J.L.M.M. Oomen, J. Blomberg, W. Genuit, G. van Velzen, J.

Chromatogr. A 892 (2000) 29

[19] J.-M.D. Dimandja, G.C. Clouden, I. Colon, J.-F. Focant, W.V. Cabey, R.C. Parry, J.

Chromatogr. A, 1019 (2003) 261

[20] J.C. Giddings, J. High Resol. Chromatogr., 10 (1987) 319

[21] The Sadtler Standard Gas Chromatography Retention Index Library, Sadtler Research Laboratories, Philadelphia 1985

[22] G.S. Cholakov, W.A. Wakeham, R.P. Stateva, Fluid Phase Equilibria 163 (1999) 21 